\title{
Prospective comparative study of dose dense neo-adjuvant chemotherapy followed by chemo-radiation and definitive chemo- radiation alone in stage IB2-IVA cervical cancer
}

\author{
Shashidhar V. Karpurmath ${ }^{1}$, Shravan Nimma ${ }^{2}$, Geeta S. Narayanan ${ }^{2}$, Manjunath I. \\ Nandennannavar ${ }^{1}$
}

\begin{abstract}
${ }^{1}$ Department of Medical Oncology, ${ }^{2}$ Department of Radiation Oncology, Vydehi Institute of Medical Science and Research Centre, Bangalore, Karnataka, India
\end{abstract}

Received: 30 July 2016

Accepted: 04 August 2016

\section{*Correspondence:}

Dr. Shashidhar V. Karpurmath,

E-mail: shashivk5@gmail.com

Copyright: (c) the author(s), publisher and licensee Medip Academy. This is an open-access article distributed under the terms of the Creative Commons Attribution Non-Commercial License, which permits unrestricted non-commercial use, distribution, and reproduction in any medium, provided the original work is properly cited.

\begin{abstract}
Background: The standard of care for locally advanced cervical cancer is chemo-radiation. Role of neo-adjuvant chemotherapy not clear yet. Dose dense, short-term neo-adjuvant chemotherapy with early initiation of radiotherapy has shown survival benefit but randomised trials not available yet. Hence this study was done for feasibility of neoadjuvant chemotherapy and for its comparison with the standard chemo-radiation.

Methods: Cervical cancer patients not randomly assigned into two arms. Arm A received 3 cycles NACT of paclitaxel and carboplatin weekly. All patients of arm A after NACT and arm B received EBRT on linear accelerator for a dose of 4500-5000cGy in 23-25 fractions with concurrent cisplatin 40mg/m 2 followed by 3 applications of HDR intracavitary brachytherapy of 700 or $800 \mathrm{cGy}$ respectively. All patients were assessed clinically and radiologically with MRI after completion of CTRT and at follow up of 6 weeks.

Results: A total of 15 patients treated in each arm. Stage IIB and IIIB constituted $93 \%$ of the patients, well matched in both the arms. The CR rate at follow up of 6 weeks after NACT was $73 \%$ compared to only $46.7 \%$ in arm B. Neutropenia of grade 3 were seen equally in both the arms and grade 2 neutropenia was seen in about $60 \%$ patients in arm A compared to only $20 \%$ patients in arm B which was manageable without growth factors.

Conclusions: Dose dense neo-adjuvant chemotherapy is a feasible and effective option in treating cancer cervix.
\end{abstract}

Keywords: Neo-adjuvant chemotherapy, Cancer cervix, Dose dense

\section{INTRODUCTION}

Cervical cancer is the fourth most common cancer among women worldwide. ${ }^{1}$ It is the second most common diagnosed cancer and third most leading cause of death in less developed countries. India accounts for $25 \%$ of cervical cancer deaths in less developed countries. Concurrent chemo radiotherapy (CRT) has become the standard of care for cervical cancer patients from stages $1 \mathrm{~B} 2$ to IVA. ${ }^{2}$ There is $6 \%$ improvement in the survival rates with the addition of chemotherapy to radiation as per the meta-analysis of 13 trials comparing chemo- radiation and radiation alone. ${ }^{3}$ There were no major advances in the treatment of cancer cervix after definitive chemo-radiation became the standard of care. There are still potential therapeutic advantages of giving chemotherapy before radiotherapy to reduce tumour size and control micro metastatic disease. A number of randomised trials have explored the use of neo-adjuvant chemotherapy (NACT) because of impressive response rates. As per the meta-analysis of neo-adjuvant chemotherapy for locally advanced cervical cancer 2003, there was no benefit for neo-adjuvant chemotherapy but the trials which used shorter cycle lengths showed $7 \%$ 
absolute improvement in 5-year survival. Results for overall and loco-regional disease-free survival and metastases-free survival similarly suggested a benefit of short cycle chemotherapy. ${ }^{4}$ The gap between the completion of chemotherapy and the initiation of radiotherapy also played an important role with early initiation of radiotherapy resulting in improvement in survival rates. So in this study, we have used lesser number of neo-adjuvant chemotherapy weekly cycles with early initiation of radiotherapy on week 4 and compared with the definitive chemo-radiation arm.

\section{METHODS}

This was a prospective, non-randomised, double arm, comparative study in cervical cancer patients undertaken to compare the neo adjuvant chemotherapy followed by definitive chemo-radiation to definitive chemo-radiation alone. 30 patients were included in this study with fifteen patients in each arm. Arm A consisted of patients on Neo-adjuvant chemotherapy followed by concurrent chemo-radiotherapy. Arm B consisted of patients receiving only concurrent chemo-radiotherapy. Patients of biopsy proven squamous cell carcinoma of cervix, between 20-60 years of age, stage IB2- IVA who were considered suitable for curative treatment were included. Patients with metastatic disease, previous history of partial hysterectomy, history of radiation to pelvis in the past and those who had contraindications for use of chemotherapy were excluded from the study. All the patients were treated with concurrent chemo-radiation. Chemotherapy was delivered along with EBRT.

\section{Chemotherapy}

Patients in arm A received injection paclitaxel $(60 \mathrm{mg} / \mathrm{m} 2)$ and injection carboplatin (AUC-2) weekly for a total of 3 cycles. Radiotherapy was started from week 4 concurrently with injection cisplatin $\left(40 / \mathrm{mg}^{2}\right)$ weekly. Patients in arm B were treated with conventional chemoradiation with Injection cisplatin $\left(40 / \mathrm{mg}^{2}\right)$ weekly, along with radiation therapy. All patients underwent CT simulation and 3D conformal planning. Patients were instructed to maintain full bladder during the acquisition of CT scan and during the treatment delivery. The treatment volumes were defined. GTV was defined as tumour+cervix+uterus. CTV $\mathrm{T}$ was defined as $1 \mathrm{~cm}$ symmetrical margin to GTV. Parametrium was not contoured separately. The part of parametrium was included in the CTV and part of it in CTV N. GTV N was defined as Lymph nodes visible on imaging and CTV N as low para-aortic, common iliac, external iliac, internal iliac and presacral lymph nodes. Inguinal nodes were included in stage III A disease. PTV was defined as CTV $\mathrm{T}+\mathrm{CTV} \mathrm{N}+\mathrm{GTV} \mathrm{N}$, with additional $5 \mathrm{~mm}$ margin.

Contouring of normal surrounding critical structures such as rectum, bladder, right and left femoral heads and small bowel were done. A four field technique with two opposing anterior-posterior and two opposing lateral portals was used. All the patients in both the groups received 3 fractions of ICBT (Intra cavitary brachytherapy) weekly. All the ICBT were done under spinal anesthesia, CT scan based point A planning was done (brachyvision software). Each cycle patient received $7 \mathrm{~Gy} /$ fraction with bowel and bladder in normal limits with HDR application.

\section{Statistical analyses ${ }^{5-8}$}

\section{Statistical methods}

Descriptive and inferential statistical analysis was carried out in the present study. Results on continuous measurements were presented on Mean \pm SD (min-max) and results on categorical measurements were presented in number (\%). Significance was assessed at $5 \%$ level of significance. The following assumptions on data were made.

\section{Assumptions}

- Dependent variables should be normally distributed.

- Samples drawn from the population should be random, Cases of the samples should be independent. Student t-test (two tailed, independent) was used to find the significance of study parameters on continuous scale between two groups (inter group analysis) on metric parameters. Leven $1 \mathrm{~s}$ test for homogeneity of variance was performed to assess the homogeneity of variance. Analysis of variance (ANOVA) was used to find the significance of study parameters between three or more groups of patients. Chi-square/fisher exact test was used to find the significance of study parameters on categorical scale between two or more groups.

\section{Significant figures}

+ Suggestive significance ( $\mathrm{P}$ value: $0.05<\mathrm{P}<0.10)$.

* Moderately significant ( $\mathrm{P}$ value: $0.01<\mathrm{P} \leq 0.05$ )

** Strongly significant $(\mathrm{P}$ value: $\mathrm{P} \leq 0.01)$

The Statistical software namely SAS 9.2, SPSS 15.0, Stata 10.1, MedCalc 9.0.1,Systat 12.0 and R environment ver.2.11.1 were used for analysis of data and Microsoft word and Excel have been used to generate graphs, tables etc.

\section{RESULTS}

The median age of patients in our study was 50 years (3770 years). All the patients in the study had squamous cell carcinoma. The patients were all belonging to stages IIA, IIB and IIIB. Most of the patients were locally advanced. Both the groups were stage matched. All the patients were examined initially. The clinical findings were 
divided into tumor primary size, parametrial extension and vaginal extent, each of the parameters were given score e.g. $0,1,2,3$, 4. After giving an individual score the three parameters were added to get a total score (min 0 to max 10). MRI pelvis with contrast was done for all the patients before treatment. The size (volume in cc) was calculated for all the patients in both the groups and was compared. As mentioned in the study, all patients in arm A received neo-adjuvant chemotherapy i.e. paclitaxel and carboplatin weekly for 3 weeks followed concurrent chemo-radiation (EBRT and weekly cisplatin). All patients in arm B received concurrent chemo-radiation only. Number of cycles of cisplatin received varied in two arms i.e. 5 cycles were received by 0 patients in arm A vs 5 Patients in arm B (0\% vs 33.3\%), 4 cycles were received by 11 patients in arm A vs 9 patients in arm B (73.3\% vs $60 \%$ ), only 3 cycles were received by 4 patients in Arm A vs 1 patient in Arm B (26.7\% vs $6.7 \%)$.

\section{Toxicities}

Grade 3 neutropenia was similar in both the groups, grade 2 Neutropenia was more in arm A compared to arm B (60\% vs $20 \%$ ) and grade 1 neutropenia was more in arm B compared to arm $\mathrm{A}(40 \%$ vs $6.7 \%)$. Few patients in both the arms had grade II-III gastro-intestinal toxicities (nausea, vomiting and loose stools). The week at which the maximum grade of neutropenia occurred varied between both the arms. In the arm A, neutropenia was noted in mostly in $2^{\text {nd }}$ and $3^{\text {rd }}$ weeks of receiving concurrent chemo-radiation i.e. during weeks 6-10 (n-11, $73 \%$ ) of overall treatment time, where as in Arm B the maximum grade of neutropenia was noted in 6 patients each in weeks 3-5 and 6-10 weeks of concurrent chemoradiation.

All the patients in both the groups were assessed clinically after the completion of concurrent chemoradiation in both the groups. More number of patients had complete response of primary tumor in arm A (n-5) compared to arm B (n-2) $(33.3 \%$ vs $13.3 \%), 13 / 14$ patients in arm A were free of parametrial induration compared to $7 / 14$ patients in arm B (86.7\% vs $46.7 \%)$, $11 / 11$ patients in arm A were free of vaginal extension compared to $13 / 13$ patients in arm B (100\% vs $86.7 \%)$. Hence all together 4 vs 1 patient in arm $\mathrm{A}$ and $\mathrm{B}$ respectively had complete response (CR). 14 patients in arm A had good response compared to 5 patients in arm B $(94.3 \%$ vs $40 \%)(\mathrm{p}<0.001)$.

Table 1: Stage of patients studied and statistically similar in two groups with $\mathbf{P}=\mathbf{0 . 8 4 7}$.

\begin{tabular}{|l|llll|}
\hline Stage & Arm A & \multicolumn{3}{c|}{ Arm B } \\
\hline II A & No & $\mathbf{\%}$ & No & \% \\
\hline II B & 7 & 6.7 & 1 & 6.7 \\
\hline III B & 7 & 46.7 & 5 & 33.3 \\
\hline Total & 15 & 46.7 & 9 & 60.0 \\
\hline
\end{tabular}

Table 2: Initial clinical findings of patients studied.

\begin{tabular}{|c|c|c|c|c|c|}
\hline \multirow{2}{*}{ Initial clinical findings } & \multicolumn{2}{|c|}{$\operatorname{Arm} A(n=15)$} & \multicolumn{2}{|c|}{ Arm B (n=15) } & \multirow{2}{*}{$P$ value } \\
\hline & No & $\%$ & No & $\%$ & \\
\hline \multicolumn{6}{|l|}{ Primary size } \\
\hline$<2 \mathrm{~cm}$ & 0 & 0.0 & 0 & 0.0 & \multirow{3}{*}{0.690} \\
\hline $2-4 \mathrm{~cm}$ & 10 & 66.7 & 11 & 73.3 & \\
\hline$>3 \mathrm{~cm}$ & 5 & 33.3 & 4 & 26.7 & \\
\hline \multicolumn{6}{|l|}{ Parametrium } \\
\hline No parametrial involvement & 1 & 6.7 & 1 & 6.7 & \multirow{5}{*}{0.612} \\
\hline One side medial $1 / 3$ rd involvement & 5 & 33.3 & 3 & 20.0 & \\
\hline Both sides medial $1 / 3$ rd involvement & 2 & 13.3 & 2 & 13.3 & \\
\hline One side till lateral pelvic wall involvement & 5 & 33.3 & 9 & 60.0 & \\
\hline Both sides till lateral pelvic wall involvement & 2 & 13.3 & 0 & 0.0 & \\
\hline \multicolumn{6}{|l|}{ Vaginal extension } \\
\hline No vaginal extension & 4 & 26.7 & 2 & 13.3 & \multirow{4}{*}{0.371} \\
\hline Upper 1/3rd vagina involved & 2 & 13.3 & 3 & 20.0 & \\
\hline Middle $1 / 3$ rd vagina involved & 7 & 46.7 & 4 & 26.7 & \\
\hline Lower $1 / 3$ rd vagina involved & 2 & 13.3 & 6 & 40.0 & \\
\hline
\end{tabular}

MRI pelvis with contrast was done for all the patients after concurrent CTRT and was compared between both the study arms. The mean percentage reduction in MRI volume in arm A was $86 \%$ compared to $78.5 \%$ in arm B $(p=0.034)$. With this it can be observed that the reduction in volume of disease was more in the neo-adjuvant arm ( $\operatorname{arm~A)~than~the~concurrent~CTRT~arm~(Arm~B).~}>90 \%$ reduction of volume was seen in 6 patients in arm $A$ compared to 3 patients in arm B, 8 patients in both the arms had $75-90 \%$ reduction in the volumes, only 1 patient 
in arm A had reduction $<75 \%$ compared to 4 patients in arm B. All the patients in both the groups received 3 fractions of ICBT (intra cavitary brachytherapy) weekly. All the ICBT were done under spinal anesthesia, CT scan based point A planning was done (brachyvision software). Each cycle patient received $7 \mathrm{~Gy} /$ fraction with bowel and bladder in normal limits with HDR application. No major toxicities or complications occurred during brachytherapy. The mean overall treatment time (OTT) in arm A was 78.73 days (min-62 days and max-94 days) vs 56.73 days (min-45 days and max-80 days). All the patients completed treatment and were asked to come for a follow up after 6 weeks from the day of discharge.

\section{Follow up}

On follow up at 6 weeks patients were clinically examined, no primary tumor was seen in 11 patients in arm A compared to 7 patients in arm B (73\% vs 46.7). All patients in arm A were free of parametrial induration whereas 3 patients still had parametrial induration in arm B (0\% vs $20 \%)$. No patient in both the groups had vaginal disease at follow up. All together 11 patients in arm A had CR compared to 7 patients in arm B (73.3\% vs $46.7 \%$ ) ( $\mathrm{p}<0.550)$. Follow up MRI's were done for all and the mean MRI volume at the time of follow up in arm A was $1.79 \mathrm{cc}$ compared to $4.23 \mathrm{cc}$ in arm B and the mean percentage reduction in the volume of disease in arm $\mathrm{A}$ was $96.3 \%$ compared to $92.3 \%$ in arm B ( $\mathrm{p}=0.009)$. 11 patients in arm A and 7 patients in arm B had reduction of $>95 \%$ volumes, 3 patients each in both the arms had reduction of $90-95 \%$ reduction in the volumes, only 1 patient in arm A and 4 patients in arm B had <90\% reduction in the volumes of disease.

Table 3: Initial total clinical score of patients studied

\begin{tabular}{|lllll|}
\hline \multirow{2}{*}{ Initial clinical score } & \multicolumn{3}{c}{ Arm A } & \multicolumn{2}{c|}{ Arm B } \\
\hline $1-2$ & No & \% & No & \% \\
\hline $3-5$ & 1 & 6.7 & 1 & 6.7 \\
\hline $6-10$ & 5 & 33.3 & 4 & 26.7 \\
\hline Mean \pm SD & 9 & 60.0 & 10 & 66.7 \\
\hline
\end{tabular}

$\mathrm{P}=0.529$, not significant, Student $\mathrm{t}$ test

Table 4: Neutropenia maximum grade of patients studied.

\begin{tabular}{|lllll|}
\hline \multirow{2}{*}{ Neutropenia max grade } & Arm A & \multicolumn{3}{c|}{ Arm B } \\
\cline { 2 - 5 } & No & $\mathbf{\%}$ & No & $\%$ \\
\hline 0 & 1 & 6.7 & 2 & 13.3 \\
\hline 1 & 1 & 6.7 & 6 & 40.0 \\
\hline 2 & 9 & 60.0 & 3 & 20.0 \\
\hline 3 & 4 & 26.7 & 4 & 26.7 \\
\hline Total & 15 & 100 & 15 & 100 \\
\hline
\end{tabular}

Table 5: Post chemo radiation total score of patients studied.

\begin{tabular}{|lllll|}
\hline Post chemo & Arm A & \multicolumn{3}{c|}{ Arm B } \\
radiation score & No & \% & No & \% \\
\hline 0 & 4 & 26.7 & 1 & 6.7 \\
\hline 1 & 10 & 66.7 & 5 & 33.3 \\
\hline 2 & 1 & 6.7 & 5 & 33.3 \\
\hline 3 & 0 & 0.0 & 4 & 26.7 \\
\hline Total & 15 & 100.0 & 15 & 100.0 \\
\hline
\end{tabular}

$\mathrm{P}<0.001^{* *}$, Significant, Fisher Exact test

Table 6: Follow up score of patients studied.

\begin{tabular}{|lllll|}
\hline \multirow{2}{*}{ Follow up score } & Arm A & \multicolumn{3}{c|}{ Arm B } \\
\cline { 2 - 5 } & No & \% & No & \% \\
\hline 0 & 11 & 73.3 & 7 & 46.7 \\
\hline 1 & 4 & 26.7 & 5 & 33.3 \\
\hline 2 & 0 & 0.0 & 2 & 13.3 \\
\hline 3 & 0 & 0.0 & 1 & 6.7 \\
\hline Total & 15 & 100.0 & 15 & 100.0 \\
\hline
\end{tabular}

$\mathrm{P}=0.550$, Not significant, fisher exact test

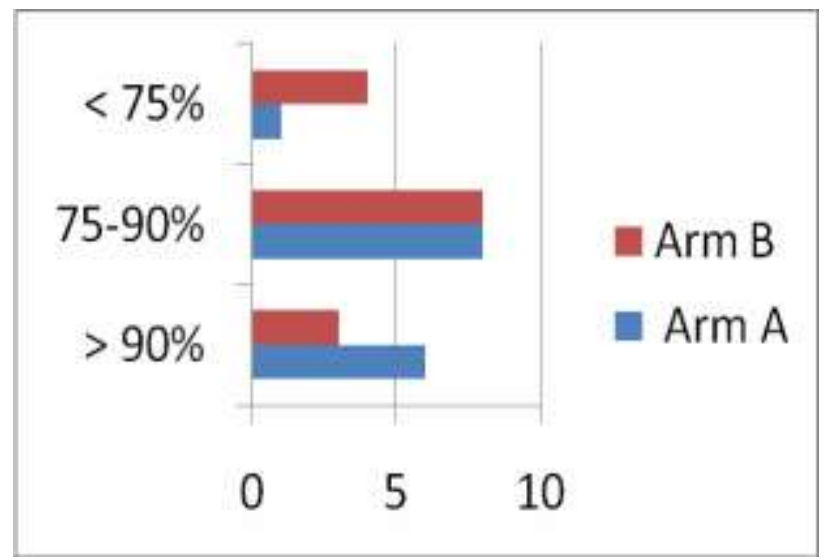

Figure 1: Bar diagram of percentage reduction in MRI vol post CTRT.

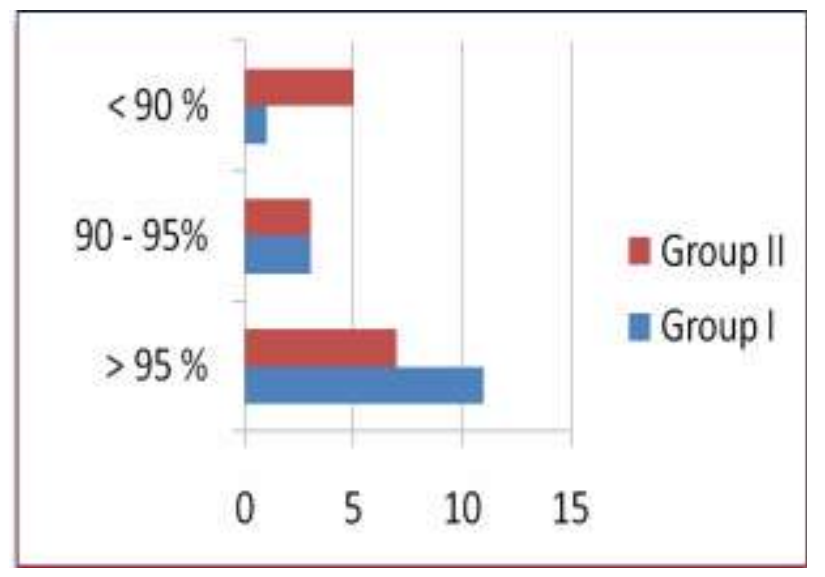

Figure 2: Bar diagram showing \% of reduction of tumour volume on MRI at follow up. 


\section{DISCUSSION}

Concept of neo-adjuvant chemotherapy in cervical cancer exists for a long time and a number of randomized trials have explored the use of neo-adjuvant chemotherapy (NACT). The first neo-adjuvant chemotherapy in cervical cancer was reported by Sardi et al in which included cisplatin, vincristine and bleomycin administered before surgery for 3 cycles resulting in improved rate of respectability. ${ }^{9}$ Neo adjuvant chemotherapy decreases the size of tumour, decreases the proportion of hypoxic cells. The fraction of tumour with lesser proliferation is also decreased by neo-adjuvant chemotherapy thus increasing the sensitivity of the tumour to radiation. ${ }^{10}$ However most of the randomised trials showed negative results in view of protracted chemotherapy courses and a long gap between completion of neo-adjuvant chemotherapy and initiation of radiotherapy. The trials which used shorter cycle lengths gave a pooled hazard ratio of 0.83 , equivalent to a $7 \%$ absolute improvement in 5-year survival as per the meta-analysis of neo-adjuvant chemotherapy in cancer cervix. The chemotherapy regimen used and the schedule of chemotherapy also varied. The CxII (McCormack et al) trial used a novel approach of administering weekly carboplatin and paclitaxel chemotherapy for six weeks followed immediately by standard CRT commencing in week seven. ${ }^{11}$ The results from this trial confirm that a short course of dose-dense weekly NACT with carboplatin and paclitaxel, followed by radical CRT, is feasible with acceptable toxicity. Hence we have taken a similar study in our setup to evaluate role of neo-adjuvant chemotherapy in cervical cancer patients with paclitaxel and carboplatin with reducing the number of neoadjuvant chemotherapy cycles to 3 instead of 6 cycles.

The response evaluation was done clinically and radiologically after completion of EBRT and at six weeks after completion of treatment. It was evident from the CXII trial that early reassessment also yielded relevant information on the response rates. $26.6 \%$ had $\mathrm{CR}$ rates in neo-adjuvant arm as opposed to $6.7 \% \mathrm{CR}$ rates in the conventional chemo-radiation arm. Fourteen patients in arm A had a good response which was defined as a total clinical score of $<2$ whereas only 6 patients had a good response in arm B (93.3\% vs 40\%). When seen radiologically, 6 patients in arm $A$ had $>90 \%$ reduction in volume of disease compared to only 3 patients in arm B. The observed CR rate at follow up of 6 weeks in arm A was $73 \%(n=11)$ in this study compared to only $46.7 \%$ $(n=7)$ in arm B. The remaining patients had a PR. Radiologically $14 / 15$ patients had $>90 \%$ reduction in Volume of disease compared to $10 / 15$ patients in arm B (93.3\% vs $66.6 \%)$.

On comparison, CXII trial had response rate of $70 \%$ after this short course of chemotherapy and $85 \%$ after CRT as assessed radiologically and an OS rate of $67 \%$ at 3 years. Park et al (2009) noted a response rate of $91 \%$ (assessed clinically and radiologically 10 days post treatment) in women with FIGO Ib2-IIb treated with 3 cycles of 10day cisplatin and paclitaxel prior to surgery.12 Similarly, Mori et al reported a response rate of $87 \%$ in 30 patients (all of whom completed all 6 weeks of NACT) with FIGO stage Ib2-IVa treated with 6 weeks of carboplatin and paclitaxel prior to surgery. ${ }^{13}$ Patients with stage III/IVa disease comprised $39 \%$ of those treated in CXII compared with $16 \%$ in the Mori et al study. Duen asGonza'lezet al reported response rates of $95 \%$ in 43 patients with FIGO Ib2- IIIb disease treated with 3 cycles of 3-weekly carboplatin and paclitaxel chemotherapy prior to radical hysterectomy and CRT. However in that study, response was assessed clinically and the planned dose of chemotherapy was higher. Hence efficacy of neoadjuvant chemotherapy arm was better than the conventional chemo-radiation arm as depicted by the results above.

In general NACT was well tolerated with no grade 4 or treatment-related deaths seen. In both the arms, grade III neutropenia was comparable (26.7\% in each arm). Grade 3-4 neutropenia of 7\% observed in the Mori et al and $15 \%$ reported by Duenas $\mathrm{G}$ et al. Grade 2 neutropenia was seen in about $60 \%(\mathrm{n}=9)$ patients in arm A compared to only $20 \%$ patients in arm $B(n=3)$. The corresponding G2 neutropenia rates were $13 \%, 16.7 \%$ and $16.2 \%$ in the McCormack et al, Mori et al and Duenas G et al, respectively. The current study has more no. of grade 2 neutropenia and less of grade 3-4 neutropenia events during NACT which was manageable without using growth factors.

As we can see in the timing of maximum neutropenia from the results above most of the maximum grade neutropenias were seen during 6-8 weeks of the treatment duration in NACT arm i.e. during 3-5 weeks of the concurrent CTRT. Most of the patients had neutropenias during 4th week of CTRT in NACT arm due to which patients were not able to receive 5 cycles of cisplatin and some patients could receive only 3 cycles of cisplatin. When compared to control arm (arm B) the maximum neutropenia timing was between weeks 3-5 and 6-8 equally i.e. 6 patients in each, hence patients in this arm could receive maximum 5 cycles of cisplatin for 6 patients and 4 cycles of cisplatin for 9 patients and only 1 patient received 3 cycles of cisplatin. Hence no patient in neo-adjuvant arm could complete 5 cycles of concurrent chemotherapy. In spite of this the response rates were better in neo-adjuvant arm.

Anaemia of grades 1-2 were seen in 5 patients in each of the study arms for which blood transfusions were done and corrected. No delay in treatment was seen due to anaemia. No patients had thrombocytopenia. Alopecia grade 1-2 was seen in arm A in around $60 \%$ patients $(n=9)$. Peripheral neuropathy of grade1 (tingling and numbness) was seen in 8/15 patients in arm A, which was manageable. Gastrointestinal toxicities (nausea, vomiting and diarrhoea) of grade 1-2 were seen equally in both the study arms $(n=6)$. 
All the patients in our study completed the planned radiation treatment. No major treatment related interruptions were seen in both the groups. The mean overall treatment time was 78.73 days (including 3 cycles of neo-adjuvant chemotherapy) for arm A and 56.73 days for arm B. However none of the patients in Arm A could receive planned 5 cycles of cisplatin, 11 patients received 4 cycles and 3 patients could receive only 3 cycles. In arm B 5, 9 and 1 patients received 5, 4 and 3cycles of cisplatin respectively.

We analyzed the impact of less number of cisplatin cycles on response in both the arms, which showed no statistical difference. There was no significant difference in the response rates for both the groups when analyzed for age (50 years and > 50 years) and stage (II and III).

\section{CONCLUSION}

Neo-adjuvant chemotherapy in carcinoma cervix plays an important role in increasing the response rates. Dose dense chemotherapy of short duration with limited number of cycles with early initiation of definitive chemo-radiation is a feasible and more effective option of treating cancer cervix. Long-term follow up and more number of patients are required to assess further role of NACT in Cervical cancer.

\section{ACKNOWLEDGEMENTS}

Authors would like to thank the entire radiation oncology and medical oncology departments of Vydehi Institute of Medical Science and Research Centre, Bangalore, India for their support.

Funding: No funding sources Conflict of interest: None declared

Ethical approval: The study was approved by the Institutional Ethics Committee

\section{REFERENCES}

1. Lindsey AT, Freddie B, Rebecca LS, Jacques F, Joannie LT, Ahmedin J. Global cancer statistics. 2012, Cancer J Clin. 2015;65:87-108.

2. Eifel PJ, Berek JS, Thigpen JT. Gynecologic Cancers. Section 2. Cancer of the cervix, vagina, vulva. In: DeVita VT Jr, Hellman S, Rosenberg SA editor(s). Cancer: Principles and Practice of Oncology. Sixth Edition, Philadelphia: LippincottRaven, 2001:1526-1556.
3. Claire V. Reducing uncertainties about the effects of chemoradiotherapy for cervical cancer: A systematic review and meta-analysis of individual patient data from 18 randomised trials. J Clin Oncol. 2008;26(35):5802-12.

4. Tierney J. Neoadjuvant chemotherapy for cervical cancer meta-analysis collaboration (NACCCMA) collaboration. 'Neoadjuvant chemotherapy for locally advanced cervix cancer'. Cochrane Database Syst Rev. 2004:14651858.

5. Bernard R. Fundamentals of Biostatistics, 5th Edition, Duxbury,2000:80-240.

6. Robert HR. Statistics in Medicine, second edition, Academic press. 2005;85-125.

7. Rao S, Richard J. An introduction to biostatistics, A manual for students in health sciences, 4th edition New Delhi: India. 2006:86-160.

8. Suresh KP, Chandrasekhar S. Sample Size estimation and power analysis for clinical research studies. J Hum Reprod Sci. 2012;5:7-13.

9. Sardi JE, Giaroli A, Sananes C, Ferreira M, Soderini A, Bermudez A, et al. Long- term follow-up of the first randomized trial using neoadjuvant chemotherapy in stage Ib squamous carcinoma of the cervix: the final results. Gynecol Oncol. 1997;67:619.

10. Park DC, Kim JH, Lew YO, Kim DH, Namkoong SE. Phase II trial of neoadjuvant paclitaxel and cisplatin in uterine cervical cancer. Gynecol Oncol. 2004;92:59-63.

11. Cormack M, Ledermann JA, Hall CMA, Symonds RP. A phase II study of weekly neoadjuvant chemotherapy followed by radical chemoradiation for locally advanced cervical cancer. J Clin Oncol. 2009;27:15.

12. Park DC, Suh MJ, Yeo SG. Neoadjuvant paclitaxel and cisplatin in uterine cervical cancer: long-term results. Int J Gynecol Cancer. 2009;19:943-7.

13. Mori T, Hosokawa K, Kinoshita Y, Watanabe A, Honjo H. Neo-adjuvant chemotherapy with weekly carboplatin and paclitaxel for carcinoma cervix. Int $\mathbf{J}$ Gynecol Cancer. 2008;18:85-9.

Cite this article as: Karpurmath SV, Nimma S, Narayanan GS, Nandennannavar MI. Prospective comparative study of dose dense neo-adjuvant chemotherapy followed by chemo-radiation and definitive chemo-radiation alone in stage IB2-IVA cervical cancer. Int J Reprod Contracept Obstet Gynecol 2016;5:2909-14. 